\title{
Bone Metastasis with Histology Different from The Primary Lung Cancer and The Skin Metastasis
}

\begin{abstract}
Introduction: It has already been reported in many types of malignant tumors that a solid metastasis can have a different histology and more aggressive state than the primary tumor.

Case presentation: We report the case of a 60-year-old smoker woman who presented with bone metastasis that histologically proved to be an adenocarcinoma. Bronchoscopy revealed a squamous cell lung cancer that - despite the different histology - was regarded as the primary tumor. Two weeks later a rapidly growing skin tumor developed that was also a squamous cell carcinoma, and was regarded as the distant metastasis of the endobronchial cancer.

Conclusions: In lung cancer it is important not to exclude the possibility of tumor dissemination even if the suspected primary tumor and the distant metastasis have different histologies.
\end{abstract}

Keywords: Lung cancer, Different histology, Metastasis, Tumor heterogeneity

\section{Moldvay Juditit, Szállási Zoltán' ${ }^{2,3}$, Puskás Rita ${ }^{4}$ Komáromi Tamás4, Fábián Katalin 4*, Kovalszky llona ${ }^{5}$ Pápay Judit ${ }^{5}$}

\section{Department of Tumor Biology, National Korányi Institute of Pulmonology, Buda- pest, Hungary
2 Children's Hospital Informatics Program at the Harvard-Massachusetts Institute of Technology Division of Health Sciences and Technology, Harvard Medical School, Boston, MA, USA
MTA-SE NAP, Brain Metastasis Research Group, Hungarian Academy of Sciences, 2nd Department of Pathology, Semmel- weis University, Budapest, Hungary
4 Department of Pulmonology, Semmel- weis University, Budapest, Hungary 1st Department of Pathology, Semmel- weis University, Budapest, Hungary

\section{Corresponding author: Katalin Fábián}

\section{झ drfabian.katalin@gmail.com} Department of Pulmonology, Semmelweis
University, 1125 Budapest, Diósárok u. 1/c,
Hungary

Tel: +36303885443

cytology showed squamous cell carcinoma that was regarded as the distant metastasis of the right central lung cancer. During her hospitalization pathologic fracture of the left humerus occurred. The general condition of the patient deteriorated rapidly and she died 5 weeks after her admission even without having the opportunity to receive palliative oncotherapy. In summary, in this patient both the primary tumor and the subcutaneous metastases were squamous cell carcinomas; however, the pelvic bone metastasis proved to be an anaplastic adenocarcinoma. Neither thoracic CT nor bronchoscopy showed any other lesion that could have been regarded as synchronous primary lung cancer or other primary tumor, such as thyroid or breast cancer. Moreover, abdominal CT did not show other lesion referring to 
extrathoracic primary tumor but only adrenal metastasis, typical metastatic site of lung cancer.

\section{Discussion}

In the presented case we might speculate that the right lower lobe endobronchial tumor contained adenosquamous elements, and during the metastatic process a tumor cell selection occurred resulting in different histology from the primary lung cancer. This is in accordance with the concept of tumor cell heterogeneity, i.e. highly metastatic cells are present as a sub-population in a primary tumor. It is well known that there are different mechanisms of clonal variation including clonal selection, parallel evolution, dynamic heterogeneity, clonal dominance and stem cell expansion [4]. In lung adenocarcinoma, the prognostic significance of KRAS mutation has already been documented [5]. The correlation between primary tumor and bone metastasis in non-small cell lung cancer was investigated by Badalian et al, who demonstrated that KRAS mutational status of the primary tumor did not predict the status of the bone metastatic tissue, since they had observed both emergence of mutant clones in metastases from wild-type primary tumors and loss of mutant clones in metastases from mutant primaries in addition to the maintained mutant status [6].
Skin metastases from lung cancer are rare with a reported incidence of 1-2\% [7]. They are more frequent in patients aged over 40 years, and are associated with a particularly aggressive biological behavior [8]. In our patient active oncotherapy, which could have contributed to an artificial selection pressure for clonal divergence or stem cell expansion was not possible to imply, therefore, this case might prove that even in the absence of therapeutic intervention histological difference between primary tumor and metastasis can be observed.

\section{Conclusion}

In our case, multiple distant metastases with different histology could be demonstrated. The skin involvement had the same squamous cell carcinoma histology as the primary lung cancer, while the bone metastasis was found to be an adenocarcinoma with a molecular feature that might be regarded as one of the most aggressive ones in the adenocarcinoma subgroup.

\section{Competing Interests}

Authors disclose no conflict of interest. 


\section{References}

de Bruin EC, McGranahan N, Mitter R, Salm M, Wedge DC, et al. (2014) Spatial and temporal diversity in genomic instability processes defines lung cancer evolution. Science 346: 251-256.

Yokota J (2000) Tumor progression and metastasis. Carcinogenesis 21: 497-503.

Cserepes M, Ostoros G, Lohinai Z, Raso E, Barbai T, et al. (2014) Subtype-specific KRAS mutations in advanced lung adenocarcinoma: a retrospective study of patients treated with platinum-based chemotherapy. Eur J Cancer 50: 1819-1828.
4 Talmadge JE (2007) Clonal Selection of Metastasis within the Life History of a Tumor. Cancer Res 67: 11471.

5 Rodenhuis S, Slebos RJ (1992) Clinical significance of ras oncogene activation in human lung cancer. Cancer Res 52: 2665s-2669s.

6 Badalian G, Barbai T, Rásó E, Derecskei K, Szendrői M, et al. (2007) Phenotype of bone metastases of non-small cell lung cancer: epidermal growth factor receptor expression and K-RAS mutational status. Pathol Oncol Res 13: 99-104.

7 Mollet TW, Garcia CA, Koester G (2009) Skin metastases from lung cancer. Dermatol Online J 15: 1.

8 http://www.dermnetnz.org/lesions/metastasis.html. 